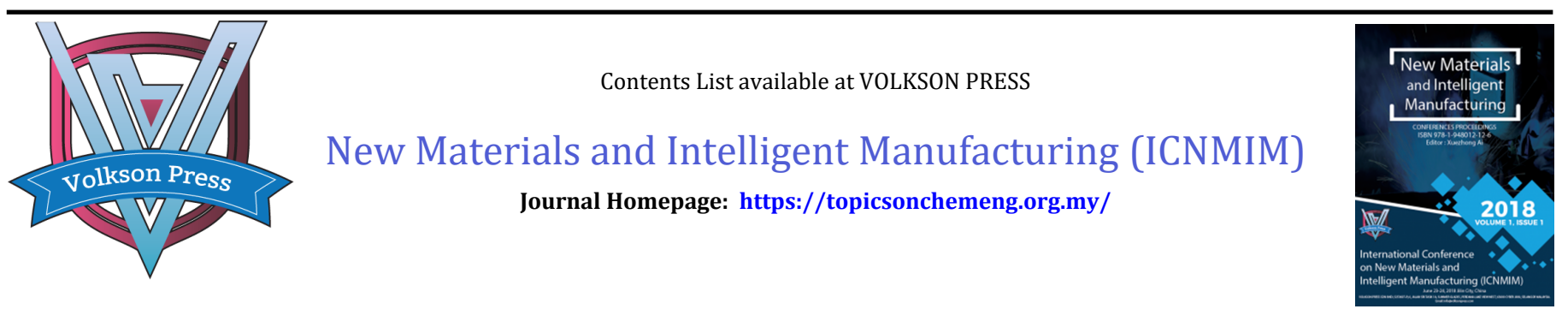

ISBN: 978-1-948012-12-6

\title{
STRUCTURAL DESIGN OF BIOMASS CONTINUOUS FERMENTATION SYSTEM
}

\author{
Hou Zhesheng* , Fang Yanzhao and You Yi \\ College of Mechanical \& Electrical Engineering, Jilin Institute of Chemical technology, Chengde Street, Jilin City, Jilin, China \\ *Corresponding Author E-mail: 13844675398@126.com
}

This is an open access article distributed under the Creative Commons Attribution License, which permits unrestricted use, distribution, and reproduction in any medium, provided the original work is properly cited

\section{ARTICLE DETAILS}

\section{Article History:}

Received 26 June 2018

Accepted 2 July 2018

Available online 1 August 2018

\section{ABSTRACT}

\begin{abstract}
A continuous biomass fermentation system, in particular an anaerobic continuous fermentation system with agricultural straw as a carbon source. After the culture medium and biomass material storage tank configuration is completed, solid-liquid ratio by controllably shaftless screw feed device into the anaerobic fermentation tank fermentation. The anaerobic fermentation tank is a flexible structure that is squeezed by external forces to achieve material mixing. Since the biomass is in the form of particles, in order to increase the fermentation efficiency, the biomass is fed into the grinding device during fermentation and re-enters the fermenter after grinding. At the end of the fermentation, most of the residue will be squeezed out of the tank and a small part will remain at the bottom of the tank as a seed. New substrate will be fed into the feeder tank, to achieve efficient continuous fermentation of biomass.
\end{abstract}

\section{KEYWORDS}

Biomass; Continuous fermentation; Anaerobic fermentation tank.

\section{INTRODUCTION}

Crop straw is a large amount of biological resources. Crop straw is composed of a large amount of organic matter and a small amount of inorganic matter and water. The main components of organic matter are cellulose-based carbohydrates, in addition to a small amount of crude protein and crude fat. Carbohydrates also consist of cellulosic substances and soluble sugars. Cellulose substances are the main components of plant cell walls and include cellulose, hemicellulose and lignin. Therefore, using straw as raw material to produce polysaccharides has a good market prospect [1-3].

Fermentation equipment that has appeared on the market can only undergo non-continuous batch fermentation due to the influence of the state of straw pellets, and the fermentation substrate and the residue cannot be automated. The metabolites of the fermentation process cannot be separated in situ and produce antagonistic effects. Moreover, it has the disadvantages of low productivity, high energy consumption, and low safety factor. In order to achieve efficient and safe production of fermentation tanks and make up for the lack of similar products on the current market, a continuous biomass fermentation system was designed and researched. Based on a study, biomass continuous fermentation system using crop straw as raw material can effectively solve these problems, and solve the environmental problems of burning straw, and also reduce resource waste [4-5]. According to research, the biomass continuous fermentation system has greatly shortened the fermentation cycle and laid the foundation for the rapid development of dairy products, beverages, bio-engineering, pharmaceuticals, fine chemicals and other industries [6].

\section{BIOMASS CONTINUOUS FERMENTATION SYSTEM}

The biomass continuous fermentation system consists of a storage tank, a feeder and a fermenter system. Under the control of the control system, a continuous fermentation process is realized.

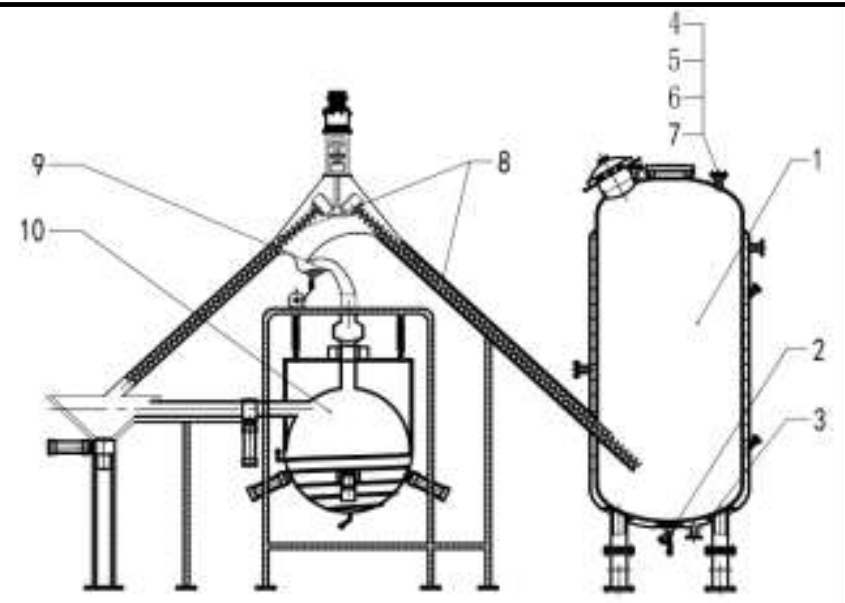

Figure 1: Biomass continuous fermentation system equipment diagram 1-Storage tanks 2-Air outlet 3-slagging port 4-Solid Injection Port 5Liquid injection port 6-Injection port 7-Air Intake 8-feeder 9-Grinding machine 10-Fermenter

\subsection{Storage tanks}

The storage tank is an ellipsoidal structure with liquid injection ports, gas injection ports, exhaust ports, and slag discharge ports. According to a group of researchers, it is also equipped with temperature sensors, pressure sensors, redox potentiometers, and $\mathrm{pH}$ meters and other electronic instruments [7-8]. The role of the storage tank is to pretreat the biomass and the culture medium so that they can meet all the requirements before fermentation.

The biomass is broken into 3-8 mm pellets, passed through a solid injection port, injected into a storage tank, and the medium is injected into 
the storage tank through the liquid injection port. Their injection volume was controlled so that the liquid medium and biomass weight ratio was 15:1. After soaking, the media removes oxygen from the biomass. According to the number of electronic instruments, the temperature, pressure, redox potential, and $\mathrm{pH}$ were adjusted to control the conditions required for microbial growth.

\subsection{Feeder}

Compared to conventional screw feeders, this feeder is a shaftless spiral structure. The middle part of the feeder is a helical spring structure with a certain pitch and the feeding spiral blade is fixed on the spiral structure. The shaftless screw is in contact with the feed pipe with rubbing strips. The feed pipe is supported by a height-adjustable structure. The feeding port of the shaftless screw feeder penetrates deep into the storage tank, and the discharge port of the shaftless screw feeder connects to the feed port of the fermenter. Based on a study, the end of the shaftless feeder is connected with the driving motor shaft, and the ratio of biomass to the medium can be controlled by adjusting the speed of the driving motor and the height of the feeding tube [9].

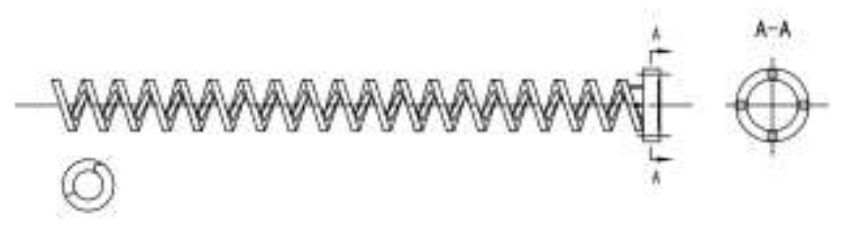

Figure 2: Feeder

\subsection{Fermentor system}

The tank of the fermenter is a spherical structure made of flexible material with a feed inlet at the top and two discharge ports at the middle and upper part. The upper part of the feed port has a pneumatic shut-off valve, a reciprocating grinder, a ball tee, a pneumatic stop valve, and a material feed inlet. The discharge port is in turn connected to the pneumatic stop valve and the ball tee. The lower half of the fermenter tank is supported by a hemispherical spiral tube structure. The spiral tube has an inlet and an outlet. The internal circulation medium controls the temperature and adjusts the temperature of the tank. The hemisphere spiral structure is fixed between the reinforcement structure and is fixedly connected with the suspension device. According to a research, the suspension device is connected with the tank body fixing bracket through the spring, and the gravity meter replaces a group of springs to achieve the weight control of the tank body [10-11]. The fixed end of the tank extruding, and deforming mechanism is fixed on the spiral structure, the movement end of the squeezing mechanism receives the control signal, and the flexible tank body is pressed together to realize the material mixing in the tank body. The inner wall of the fermentation tank is a membrane separation layer, and the membrane separation layer is intertwined with the flexible tank material. The micro-channel collects the filtrate after the membrane filtration, and then passes through the filtrate outlet and the negative pressure device enters the filtrate separation system.

\section{OPERATIONAL PROCESS}

The biomass is broken into 3 to $8 \mathrm{~mm}$ pellets, passed through a solid injection port, injected into a storage tank, and the medium is injected into the storage tank through the liquid injection port. Their injection volume was controlled so that the liquid medium and biomass weight ratio was 15:1. After soaking, the media removes oxygen from the biomass.

The feeder works, and a certain proportion of liquid medium and biomass are sent to the grinding device. After the grinding device grinds, the material is sent to the fermenter.

The can body extrusion mechanism presses the can body according to the control process to achieve mixing. Due to the internal degradation of the biomass, during the fermentation process, the biomass can be pressed into the material outlet by the squeezing device at the same time and reach the ball outlet. The driving motor starts, the clutch connection alternative no shaft feeding system feeding into the three-way ball. Re-enter the fermenter through the reciprocating grinding device and ferment again. After completion of the fermentation process, all the pressing means is operated to achieve product into the discharge port through the stop valve, the material reaches the three-way ball. The pneumatic stop valve opens, and the product is discharged from the discharge opening. When the degraded material is discharged, the feed inlet and discharge valve are closed to ensure fermentation conditions. Most of the biomass after discharging the tank, shut-off valve to close the discharge port, the remaining portion not excluded, may be left in the fermentation tank as a seed, starts feeder into a new medium, the next cycle of fermentation, to achieve a continuous fermentation [12]

\section{CONCLUSION}

Biomass continuous fermentation system is a biomass-based fermentation system with no dead spots that can be separated in-situ. Storage tanks, feeders and fermenters can be continuously fermented under the control of the control system. The substrate needed by the fermenting microorganisms is controlled in the tank and reaches the basic conditions for fermentation such as $\mathrm{pH}$, temperature, oxidation-reduction potential, moisture, and trace element content. The feeder achieves a solid-liquid ratio-controlled biomass transport. No spherical stirred tank fermenter design, preparation of flexible silicone rubber material, to minimize open, mouthpiece multiplexing functions. The sensor is not installed in the tank as much as possible, or only the surface of the tank is installed so that the tank can have no dead ends. Followed by kinetic system, which uses a mechanical structure to simulate peristaltic motion and acts on the outer wall of a flexible fermenter. Through the denaturation of the external wall of the local tank, it acts on the internal materials of the tank to achieve the mixing effect, instead of the traditional stirring method, it cannot pollute the internal environment, and does not cut the mycelium, and the mixture is soft. Due to the membrane separation device inside the tank, the metabolites can be separated to make the degradation of the substrate more efficient. For co-cultivation of microorganisms, metabolites are separated in time, competition is not easy to occur, and target product-oriented symbiosis culture is easy to achieve. Eliminating negative effects due to the peristalsis multi brought inside wall fouling and concentration polarization phenomena, fermentation of biomass in a particulate form, without completely grinding, mid-fermentation fermented straw particles re-entering the fermenter again after grinding. At the late stage of fermentation, most of the substrate exits the fermenter and remains partially in the tank to act as seeds for continuous fermentation.

\section{ACKNOWLEDGMENTS}

Fund Project : Significant Scientific and Technical Project in Jilin (20170201003GX)

\section{REFERENCES}

[1] Chen, Y.H., Tian, F.Y., Yan, Y.F. 2018. Current status, existing problems and development proposals of crop straw comprehensive utilization. Chinese Journal of Agricultural Mechanization, (2), 67-73.

[2] Liang, W., Nie, Y. 2017. Comprehensive Utilization of Crop Straw: Foreign Experience and China's Countermeasures. World Agriculture, (9), 34-38.

[3] Gao, X., Xu, K.X. 2017. Study on Comprehensive Utilization of Crop Straw in Anhui Province. Journal of Anhui Agricultural Sciences, (28), 5557.

[4] Hou, Z.S., Tong, J. 2007. Bionic Coupling Design of Peristaltic Fermentors. Journal of Agricultural Machinery, 38 (6), 65, 100-102

[5] Abdeen Mustafa Omer. 2018. Renewable for Power Generation: Clean Energies for Sustainable Development and Environment. Energy Reviews, 2 (1), 14-19.

[6] Jia, B.X. 2018. A review of new bio-pharmaceutical fermentation tanks. Jiangsu Science \& Technology Information, (3), 35-37.

[7] Li, J.H., Yu, Y.Y., Wang, C.X. 2006. Development and application of horizontal solid material fermentation tanks. Feed Industry, (13), 5-6.

[8] Liu, A.Q., Fang, S.J., Li, L. 2009. Structural design of GBR-03 selfcontrolled low-pressure fermentation tank. Light Industry Machinery, (1), 98-101.

[9] Blakebrough, N. 2013. Fundamentals of fermenter design. Pure and Applied Chemistry, 36 (3), 305-316.

[10] Niu, C., Xu, D.W., Wang, J. 2008. Design of fermentation tank temperature control system. Automation and Instrumentation, (5), 10-12.

[11] Shi, D., Gao, S., Lin, G.Q. 2018. Temperature Field Analysis of an Anaerobic Fermentation Tank Based on ANSYS_Shi Jiong. Journal of Jilin Institute of Chemical Technology, 35 (1), 18-22.

[12] Hou, Z.S., Tong, J., Ma, Yi, H., Tian, A.H., Chen, Y.F., Hou, M.M., Ren, L.Q. A continuous biomass fermentation system-application disclosure. Patent. 